\title{
EFFECT OF EXCIMER LASER IRRADIATION OF BIODEGRADABLE POLYMER ON ITS CHEMICAL BONDING
}

\author{
Paper M1306
}

\author{
Shan-Ting Hsu, Huade Tan, Y. Lawrence Yao \\ Department of Mechanical Engineering, Columbia University \\ New York, NY, 10027, USA
}

\begin{abstract}
The biodegradable polymer such as poly(L-lactic acid) (PLLA) is promising in drug delivery applications because its chemical structure allows it to hydrolyze into non-toxic substances in the human body. In such applications, drugs are embedded in a polymer matrix and released at the rate at which it degrades. PLLA degradation rate of is a strong function of its crystallinity. Thus, control over crystallinity allows for continuous modification of drug release profiles. The excimer laser is used in this study to induce surface crystallinity changes because its spatially uniform intensity profile is favorable for surface treatment. The effects of excimer laser irradiation on the surface morphology, crystallinity, and chemical modifications are investigated via optical microscopy, wide-angle X-ray diffraction, and X-ray photoelectron spectroscopy. A model is developed to numerically examine the spatial and temporal temperature profiles, as well as the amount of chemical modifications. It is found that PLLA crystallinity decreases as a function of laser fluence, and that the amount of chemical modifications is minimized by annealing before laser treatment and reducing laser fluences, which decrease free radical mobility and thus the dissociation quantum yield. A working window is demonstrated in which PLLA crystallinity decreases with no measurable chemical modifications, and the working window can be enlarged by decreasing free radical mobility.
\end{abstract}

\section{Introduction}

Biodegradable polymers have been attracting wide attention due to their biocompatibility and biodegradability. Being biodegradable, poly lactic acid (PLA) is used in food packaging and tissue engineering. Its chemical structure also makes it favorable in drug delivery as it hydrolyzes in the human body into lactic acid, which is then excreted showing no toxicity. In drug delivery, drugs are embedded in a polymer matrix and released as it degrades. The advantages of using biodegradable polymers in drug delivery system can be manifested by the controlled drug release profiles against time [1]. However, due to the induction period of polymer degradation caused by the time for water molecules to penetrate into the matrix, embedded drugs cannot release at the designed rate in the early stage.

Efforts have been made to modify the degradation rate of PLA. Zhao et al. [2] modified its degradation rate by adjusting temperature and $\mathrm{pH}$ value in the degradation environment. This attempt is limited since both parameters are invariants in the human body. Blending PLA with another biodegradable polymer, poly(glycolic acid), to adjust the degradation rate is also investigated [3], while the desired properties of PLA may be altered in the copolymer. Tsuji [4] investigate the effects of tacticity, content of L-lactide unit, and blending of PLLA and PDLA on the degradation rate of PLA. Although the PDLLA film can have the highest degradation rate, synthesizing PDLLA with specific ratio of PLLA and PDLA could be a tedious work.

A way to modify the degradation rate of PLA free from the above limitations is to change its crystallinity [5]. Tsuji and Ikada [6] proposed that PLA degradation begins in the amorphous region between lamellae, followed by the disorientation of the lamellae and disappearance of the spherulitic structure. Less crystalline regions are expected to degrade faster. Adjusting crystallinity is a relatively simple process, and can be accomplished through thermal treatments such as annealing, melting and quenching [7]. However, they affect the overall properties within the bulk and have limitation since accelerating the initial degradation may only require decreasing surface crystallinity while keeping the bulk intact. Recently, laser irradiation as a heating source to modify the surface crystallinity has drawn increasing attention. It is also attractive due to flexibility, ease of use, and spatial controllability.

Using the Nd:YAG laser with the photon energy lower than PLLA bond energies, Bhatla and Yao [8] reduced PLLA crystallinity, and quantified it by the wide angle X-ray diffraction (WAXD) measurements. However, the non-uniform laser spatial intensity profile makes it difficult to achieve a uniform surface treatment. Because of the incoherent light generated by the excimer laser, the beam can be homogenized without 
interference, and the homogenized beam is spatially uniform and favorable for surface treatment. Using the excimer laser, Lazare and Benet [9] treat poly(ethylene terephthalate) (PET) films, and demonstrate the presence of a thin amorphous surface layer. Lazare and Benet report that photochemical reactions are possible in addition to photothermal effects, since excimer lasers operate at UV wavelengths with photon energies higher than certain bond energies in polymers. In another attempt to decrease PET film crystallinity using the excimer laser [10], the possible chemical changes are studied via $\mathrm{X}$-ray photoelectron spectroscopy (XPS). It is found that low fluences cause very little chemical changes. These chemical changes include the dissociation of ester bonds and reduce of the number of oxygen atoms due to the emission of small molecules [11]; these modifications could alter the original properties of the polymers.

The primary objective of this work is to reduce crystallinity of PLLA films with excimer laser surface treatment, while minimizing the chemical modifications. The optical microscopy, WAXD, and XPS measurements are utilized to study the morphology, degree of crystallinity, and chemical modifications, respectively. A 2D model considering the thermal and chemical effects is proposed to numerically examine the spatial and temporal temperature profiles and the amount of chemical changes. The effects of radical mobility in the PLLA films and laser fluence have also been considered.

\section{Background}

\section{Melting and Crystallization}

Polymer melting is an amorphization process in which polymer chains detach from crystals, and crystal dimension decreases longitudinally and transversely [12]. As a rapid process, chain detachment and crystallinity decrease can occur within nanoseconds [13]; melting polymers by a nanosecond laser is thus feasible. The melting temperature $T_{m}$ of PLLA is between $170^{\circ} \mathrm{C}$ to $180^{\circ} \mathrm{C}$. Due to the difference in the fold surface energy $\sigma_{e}$ and lateral surface energy of crystals, thicker crystals are more thermodynamically stabile and thus have higher $T_{m}[14,15]$ :

$$
T_{m}=T_{m}^{0}\left(1-\frac{2 \sigma_{e}}{\Delta h_{f} l}\right)
$$

where $T_{m}^{0}$ is the equilibrium melting temperature, $\Delta h_{f}$ is the fusion heat, and $l$ is the crystal thickness. Given sufficient chain mobility, lamellar crystals thicken to reduce surface energy. Annealing enhances chain mobility, and can increase $l$ and $T_{m}$ by $5-10^{\circ} \mathrm{C}$ for PLLA $[7,16]$. Temperatures higher than $200^{\circ} \mathrm{C}$ can cause thermal degradation over minutes.

Crystallization begins with the nucleation process. Nucleation of PLLA occurs over minutes. PLLA crystal growth is given by the non-isothermal crystallization kinetics

$$
\theta(t)=1-\exp \left\{-\left[\int_{0}^{t} K(T(\tau)) d \tau\right]^{m}\right\}
$$

where $\theta(t)$ is the degree of crystallinity, $t$ is crystallization time, $K(T(\tau))$ is the reaction rate constant, and $m$ is Avrami exponent. For crystal development $t$ is also on the order of minutes. A cooling rate higher than $50{ }^{\circ} \mathrm{C} / \mathrm{min}$ prevents molten PLLA from recrystallization [17]. Since laser treatment can induce an extremely high cooling rate, recrystallization during the cooling process of laser treatment does not occur [8].

\section{Interaction between Photons and Polymers}

Polymer absorption of photons with energies exceeding the bond energy can break the bonds. A series of events is given in Fig. 1 [18]. The photons

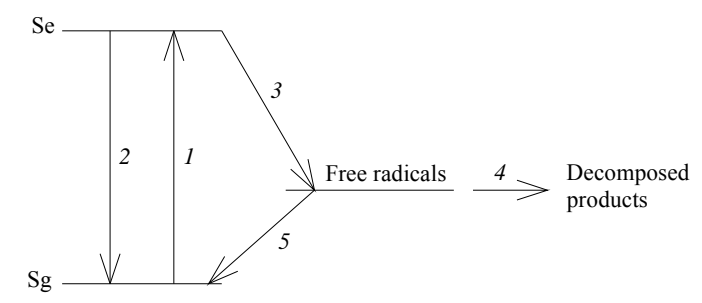

Figure 1: Elementary events following the polymer absorption of photons with energies higher than the polymeric bond energy.

excite electrons from the ground state $S g$ to the exited state Se, Event 1. Part of the energy is relaxed, Event 2; part of it breaks the bond, forming free radicals, Event 3. Some radicals generate decomposed products via Event 4, while some recombine, Event 5. Radical reactions release heat. Without oxidation, UV irradiation on PLA breaks the $\mathrm{CO}$ bonds in its backbone, separate the $\mathrm{CH}_{3} \mathrm{CHCOO}$ units, and generates free radicals R1 and R2 following Eq. (3) [19],

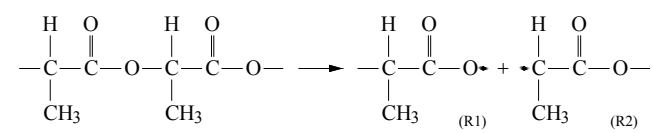


This reaction occurs between two $\mathrm{CH}_{3} \mathrm{CHCOO}$ units. As the reaction continues, the number of scissions increases, which decreases the molecular weight and number of connected $\mathrm{CH}_{3} \mathrm{CHCOO}$ units. $\mathrm{R} 1$ and $\mathrm{R} 2$ can generate smaller molecules such as $\mathrm{CO}$ and $\mathrm{CO}_{2}$ via further reactions [20]. The reactions last for microseconds after the nanosecond UV laser pulse [21]. After laser treatment, bubbles can be observed on the polymer surface $[20,22,23]$.

\section{Dissociation Quantum Yield}

Competition of Events 4 and 5 in Fig. 1 determines the dissociation quantum yield $\phi$, which is the number of dissociations occurring per photon absorbed, defined by

$$
\phi=\frac{w}{M_{n}^{0}} \frac{d\left(M_{n}^{0} / M_{n}-1\right)}{d\left(N_{p h} t\right)}
$$

where $w$ is the sample weight, $M_{n}^{0}$ is the initial molecular weight, $M_{n}$ is the molecular weight at time $t, N_{p h}$ is the number of absorbed photons per unit time. The number of scissions per chain, $\left(M_{n}^{0} / M_{n}-1\right)$, increases with radical mobility based on the cage effect. The quantum yield in the mobile state is generally larger by an order under UV irradiation [24], and is proportional to the dissociation reaction rate constant $K$ through [25]

$$
K=N_{p h} \sigma(\omega) \phi
$$

where $\sigma(\omega)$ is the photon absorption cross section with photon frequency $\omega$.

\section{Numerical Model}

To numerically investigate the spatial and temporal distribution of temperature and the amount of chemical modifications, a 2D thermal and chemical model is developed. Since PLLA is semitransparent at $\mathrm{UV}$, laser irradiation energy is absorbed by the bulk, and the bulk temperature is governed by the heat equation

$$
\rho C_{p}(T) \frac{\partial T}{\partial t}=\nabla \cdot(k \nabla T)+q(z, t)+q_{r a d}
$$

where $\rho$ is density, $C_{p}(T)$ is specific heat, $k$ is conductivity, $z$ is laser irradiation depth, $q(z, t)$ is the laser power density, and $q_{\text {rad }}$ is the reaction of radicals release heat as will be determined in Eq. (12). For an incompressible material, change in $C_{p}(T)$ during the phase transition can be approximated by
$\Delta C_{p}=\Delta h_{f} / T_{m}$, where $\Delta h_{f}$ is the heat of fusion. The specific heat is calculated as

$$
C_{p}(T)=C_{p 0}+\Delta C_{p} U\left(T-T_{m}, \Delta T\right)
$$

where $C_{p 0}$ is the specific heat in the solid state, 1000 $\mathrm{J} /(\mathrm{kg} \cdot \mathrm{K}), U$ is a step function whose value is 0 for $T \leq T_{m}-\Delta T$ and 1 for $T \geq T_{m}+\Delta T . \Delta T, 10^{\circ} \mathrm{C}$, is half the transition temperature span. Laser power density, $q(z, t)$, is a function of space and time

$$
\begin{aligned}
q(z, t)=A \cdot q_{0} \cdot & \exp (-\alpha z) \\
\cdot & \exp \left[-4 \ln 2\left(\frac{t-2 t_{p}}{t_{p}}\right)^{2}\right]
\end{aligned}
$$

where $A$ is the absorptivity, $0.07, \alpha$ is the absorption coefficient, $10^{3} \mathrm{~cm}^{-1}$ [26], $t_{p}$ is the pulse width, $25 \mathrm{~ns}$, and $q_{0}$ is the peak power density obtained by $q_{0}=\alpha F / t_{p}$ with $F$ the laser fluence. Convection and radiation are considered on the boundaries while insulation is specified along the symmetry line. The initial temperature is set $20^{\circ} \mathrm{C}$. Change of crystal size due to annealing is not of interest, while its effect on $T_{m}$ is considered by assuming different $T_{m}$ 's for the non-annealed films $\left(175^{\circ} \mathrm{C}\right)$ and annealed $\left(180^{\circ} \mathrm{C}\right)$ films. From the discussion of crystallization and cooling rate in Sec. 2.1, it is assumed that no crystallization occurs during cooling; the assumption will be validated by our simulation results in Sec. 5.2. Crystallinity decreases as the material melts; therefore, the crystallinity change is related to the melting depth in the film.

The chemical modifications occur via Eq. (3) if R1 and R2 do not recombine. The amount of modifications depends on the number of scissions, Eq. (4). As scission occurs, the connections of the $\mathrm{CH}_{3} \mathrm{CHCOO}$ units at their ester group with another unit are broken, and the number of such connected $\mathrm{CH}_{3} \mathrm{CHCOO}$ unit decreases. Therefore, the number density of the $\mathrm{CH}_{3} \mathrm{CHCOO}$ units with connection at their ester group to another unit is modeled. For incompressible materials, the number density is governed by the mass conservation equation

$$
\frac{\partial c}{\partial t}+\boldsymbol{u} \cdot \nabla c=\nabla \cdot(D \nabla c)+r
$$

where $c$ is the number density and normalized to be $100 \mathrm{~mol} / \mathrm{m}^{3}$ as the initial value $c_{0}, \boldsymbol{u}$ is the velocity vector, $D$ is the diffusion coefficient, and $r$ is the reaction rate. Velocity and diffusion gradients are assumed to be negligible during laser treatment, and thus $\partial c / \partial t=r$. The reaction rate is given by the degree of crystallinity $\theta$, 


$$
r=\theta \cdot r_{c}+(1-\theta) \cdot r_{a}
$$

where $r_{c}$ and $r_{a}$ are the reaction rates of the crystalline and amorphous domains, respectively. The photochemical reaction rate is proportional to the number density [27]. Thus, $r_{c}=c \cdot K_{c}$ and $r_{a}=c$. $K_{a}$ where $K_{c}$ and $K_{a}$ are the reaction rate constants of the crystalline and amorphous domains, respectively. Temperature dependence of reaction rate is expressed by Arrhenius expressions for the reaction rate constant [26]; the proportionality between the reaction rate constant and quantum yield [25] is also considered.

$$
K_{i}=K_{0} \cdot \exp \left(-\frac{E}{R T}\right) \cdot \phi_{i}
$$

where $i=c$ or $a$, and $K_{0}$ is the pre-exponential factor of the Arrhenius expression, assumed $10^{7} 1 / \mathrm{s}, E$ is the activation energy, assumed $25 \mathrm{~kJ} / \mathrm{mol}$, and $R$ is the universal gas constant. $\phi_{c}$ and $\phi_{a}$ are the step functions accounting for the quantum yields in solid and mobile states. $\phi_{c}$ is assumed 1 if $T>T_{m}$ and 0.1 if $T \leq T_{m}$ and $\phi_{a}$ is assumed 1 if $T>T_{g}$ and 0.1 if $T \leq T_{g}$ to consider the one-order-of-magnitude difference in the mobile and solid states [24]. Temperature is imported from the thermal analysis. It is assumed that this photochemical process lasts for $t_{d e g}=20 \mu \mathrm{s}$ and that no flux across the boundaries. Reaction of radicals release heat $q_{\text {rad }}$, considered as

$$
q_{\text {rad }}=\left(\frac{c_{r a d}}{2}\right)\left(\frac{H_{r a d}}{t_{d e g}}\right) U_{r a d}
$$

where $c_{\text {rad }}$ is the number density of the free radicals to be recombined or react, which is $2 c$ if all molecules in the ground state can be excited to generate free radicals (Event 1 in Fig. 1). Also in Eq. (12), $H_{\text {rad }}$ is the heat by each event, around $600 \mathrm{~J} / \mathrm{mol}$ [29], and $U_{\text {rad }}$ is the step function whose value is 1 if $t \leq t_{\text {deg }}$ and 0 if $t>t_{d e g}$ to consider the time period of the photochemical process. The reaction heat is then output to the thermal analysis. Part of the crystalline chain becomes amorphous during melting, while its motion could be constrained by surrounding crystals. Thus, at the same $T$, the initial crystalline domain is assumed to have the same mobility and reaction rate before and after melting. Since the amount of chemical modifications on the polymer chains induced via Eq. (3) is of interest and the generated free radicals only react with other radicals instead of the polymer chains [20], the subsequent chemical reactions and products are not considered in the simulation.

The coupled partial differential equations (6) and (9) with boundary and initial conditions can be solved through the finite element method. The software COMSOL Multiphysics 4.1 is used. A 2D symmetric model is developed and composed of thermal and chemical analyses. Its domain is $0.7 \mathrm{~mm} \times 20 \mu \mathrm{m}$ with laser beam size $0.5 \mathrm{~mm}$. The thermal analysis is realized by the Heat Transfer in Solids Interface of the Heat Transfer Module. The chemical analysis is accomplished by The Transport of Diluted Species Interface of the Chemical Reaction Engineering Module, which is able to compute the number density of a single species under varying reaction rates. The COMSOL smoothed Heaviside function, flc2hs, is used as the step functions.

\section{Materials and Methods}

PLLA granules were provided by PURAC and used as received. The inherent viscosity of PLLA in chloroform at $25^{\circ} \mathrm{C}$ is $1.6 \mathrm{dl} / \mathrm{g}$, and the molecular weight is $56,274 \mathrm{~g} / \mathrm{mol}$ [30]. Films were prepared by solvent casting method described in [31]. The film thickness is $20-25 \mu \mathrm{m}$. Dissociation quantum yield changes with radical mobility, which can be reduced by higher crystallinity. Annealing increases crystallinity, and can obtain films with lower radical mobility when radicals are formed due to laser irradiation. Non-annealed films are termed films with high radical mobility. Annealing is conducted at $140^{\circ} \mathrm{C}$ for 1 hour. A $\mathrm{KrF}$ excimer laser with wavelength $248 \mathrm{~nm}$ and pulse width $25 \mathrm{~ns}$ is used. The homogenized laser beam has spatially uniform intensity favorable for a uniform surface treatment. The film is radiated by a single pulse with fluence 1.8 $\mathrm{J} / \mathrm{cm}^{2}$ to $2.8 \mathrm{~J} / \mathrm{cm}^{2}$ in argon atmosphere with the flow rate of 15 standard cubic feet per hour to prevent oxidation. Morphology of PLLA films is observed via optical microscopy. WAXD measurement is accomplished using the Inel X-ray diffractometer. Monochromatic $\mathrm{CuK} \alpha$ radiation with wavelength $\lambda=0.15418 \mathrm{~nm}$ at $40 \mathrm{kV}$ and $30 \mathrm{~mA}$ is used. X-ray photoelectron spectroscopy (PHI 5500 ESCA) is used to analyze chemical constituents of pre- and post-treated films. The $\mathrm{O}_{1 \mathrm{~s}}$ and $\mathrm{C}_{1 \mathrm{~s}}$ spectra are captured, and the take-off angle is $45^{\circ}$.

\section{Results and Discussion}

\section{Effects of Laser Irradiation on Morphology}

Typical morphologies of the laser treated films are given in Figs. 2(a) and 2(b). Higher fluence irradiation generates appreciable bubbles since laser energy penetrates into the bulk with low absorption coefficients. This phenomenon has also been reported in poly(methyl methacrylate) (PMMA) under excimer laser irradiation [30,31]. Its absorption coefficient is 
on the same order as PLLA $\left(10^{3} \mathrm{~cm}^{-1}\right)$, and the fluences of several $\mathrm{J} / \mathrm{cm}^{2}$ generate bubbles in both materials. Previous studies treat PET with fluences only several $\mathrm{mJ} / \mathrm{cm}^{2}[8,9]$. The difference comes from the fact that PET has high absorption coefficient $\left(10^{5}\right.$ $\left.\mathrm{cm}^{-1}\right)$, and most energy is absorbed by and concentrated on the surface, which causes lower amorphization and ablation fluences.

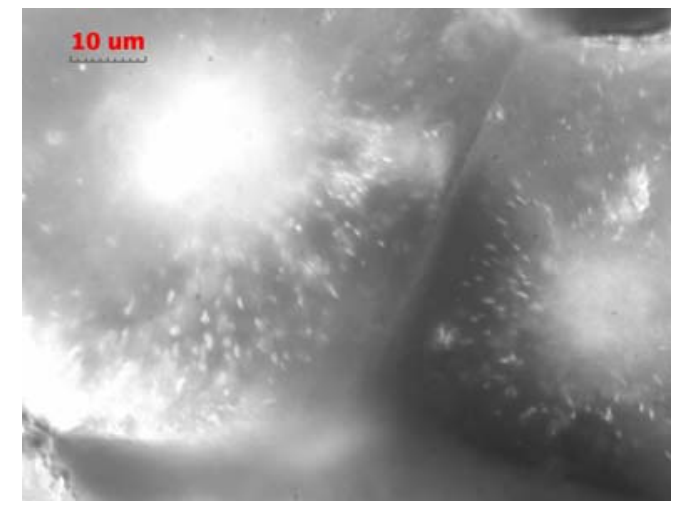

(a)

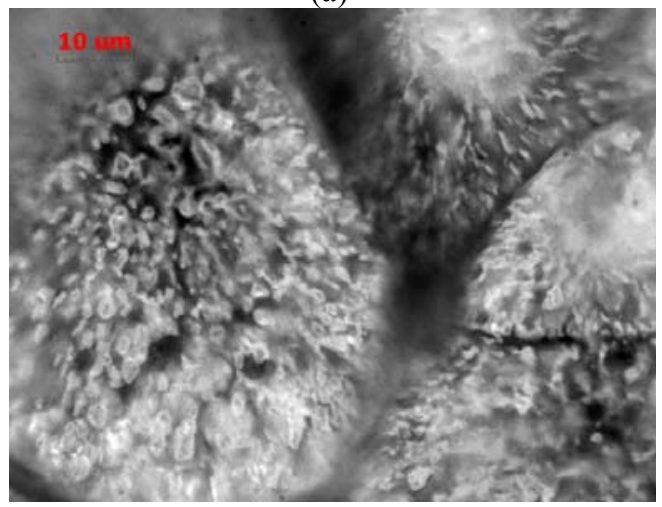

(b)

Figure 2: Morphologies of the laser treated films with low radical mobility treated with the fluence of (a) $2.6 \mathrm{~J} / \mathrm{cm}^{2}$ and (b) $2.7 \mathrm{~J} / \mathrm{cm}^{2}$.

Different explanations of bubble formation have been proposed. Srinivasan [32] suggests that the bubbles are the gas trapped by the melted and re-solidified material, while others believe that the bubbles are the gaseous products from decomposition [30]. The origin of bubble formation can be revealed by comparing the morphology shown in Fig. 2 and the XPS results shown in Figs. 7 to 9. (The XPS measurements and chemical modifications will be discussed in more detail later) Similar morphologies are obtained for the films with high radical mobility. However, for such films the bubbles are already appreciable if the fluence achieves $2.6 \mathrm{~J} / \mathrm{cm}^{2}$, while no appreciable bubbles occurring when treated by 2.5 $\mathrm{J} / \mathrm{cm}^{2}$. The results agree with the XPS measurements, which show that appreciable chemical modifications occur at $2.6 \mathrm{~J} / \mathrm{cm}^{2}$ and $2.65 \mathrm{~J} / \mathrm{cm}^{2}$ for the film with high and low radical mobility, respectively. It is believed that bubble formation is a chemical process involving decomposition of the original material. The components in the bubbles could be small molecules such as $\mathrm{CO}$ and $\mathrm{CO}_{2}[20]$.

\section{Decrease of Crystallinity by Laser Irradiation}

Crystallinity is investigated via WAXD, and the profiles for the films with high radical mobility are given in Fig. 3. The peaks become less prominent for the films treated with higher fluences, suggesting a lower crystallinity. Similar results are obtained for the films with low radical mobility, which have more prominent peaks due to more crystalline domain caused by annealing. The degree of crystallinity is calculated based on [33] with results given in Fig. 4. As expected, annealing results in a higher degree of crystallinity in the film with low radical

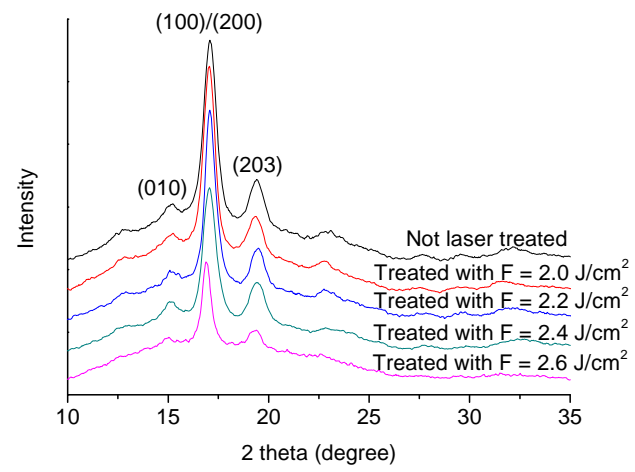

Figure 3: WAXD profiles of crystalline PLLA films with high radical mobility. Note that the peaks decrease at higher fluences (2.4 and 2.6 $\mathrm{J} / \mathrm{cm}^{2}$ ). The same trend is observed for the films with low radical mobility.

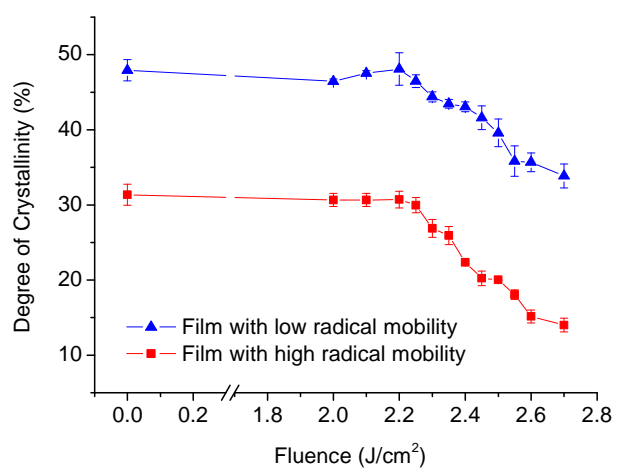

Figure 4: Crystallinity of PLLA films derived from the WAXD results as a function of laser fluence. 
mobility. Laser treatment reduces crystallinity for both films after certain fluences, which reflects that the crystallinity decrease is a thermal behavior involving polymer melting.

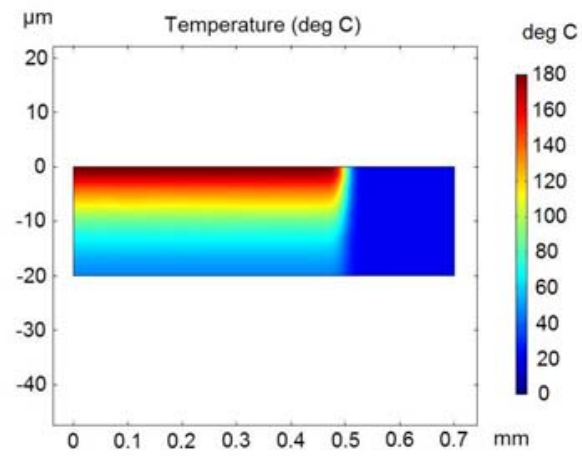

Figure 5: Simulation result of the typical temperature distribution in the film. This simulation depicts the film with high radical mobility $1 \mu$ s after the onset of a $2.4 \mathrm{~J} / \mathrm{cm}^{2}$ laser pulse.

Annealing can increase $T_{m}$ by several degrees [7]; however, the crystallinities of the two kinds of films both begin to decrease at $2.3 \mathrm{~J} / \mathrm{cm}^{2}$ (Fig. 4). Namely, the change of $T_{m}$ due to annealing does not affect the onset of crystallinity decrease. This is because the temperature increment caused by laser irradiation with different fluences is larger than the $T_{m}$ difference caused by annealing. The $T_{m}$ difference, on the other hand, causes different amount of crystallinity change. For the film with high radical mobility (the non-annealed film), the crystallinity decreases from $31.36 \%$ to $14.01 \%$ after treated with a $2.7 \mathrm{~J} / \mathrm{cm}^{2}$ pulse, which is $55 \%$ decrease. For the film with low radical mobility (the annealed film), the degree of

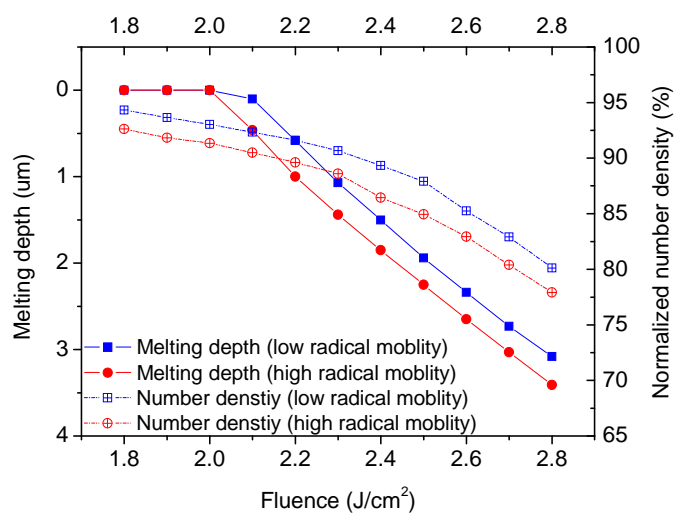

Figure 6: Simulation results of the melting depth in the film and the normalized number density of the $\mathrm{CH}_{3} \mathrm{CHCOO}$ units connecting at their ester group with other $\mathrm{CH}_{3} \mathrm{CHCOO}$ units. crystallinity decreases from $47.92 \%$ to $33.85 \%$ under the same condition, which accounts for $29 \%$ decrease. Less amount of material is melt in the annealed film. Since the WAXD measurement measures the degree of crystallinity in the bulk because of the high penetration depth of X-ray, a possible scenario is that, due to a higher $T_{m}$ in the annealed film, the melting depth is smaller and the amount of crystallinity decrease is thus smaller.

To numerically investigate the temperature distribution and melting depth in the film, a 2D thermal model is developed. A typical simulation result of the spatial temperature distribution is given in Fig. 5. The highest temperature occurs on the surface due to highest local laser energy density, and the temperature decreases with depth. Since the excimer pulse intensity is spatially uniform, the temperature distribution is not a function of the distance to the beam center. Based on the simulated temperature distribution, the melting depth is determined as given in Fig. 6. For both kinds of films, the simulation shows that melting begins from the surface once the fluence reaches $2.1 \mathrm{~J} / \mathrm{cm}^{2}$. The melting depth increases with fluence thereafter. The threshold behavior of the onset of melting agrees with the WAXD measurements, and numerically demonstrates that crystallinity change is a thermal process (melting). It is simulated that both films begin to melt at the same fluence, as agrees with the WAXD results. Since the small difference in $T_{m}$ of both films does not affect the onset of surface melting, the crystallinity decreases in both films under the same laser fluence. Simulated temperature distribution also suggests that the onset of crystallinity decrease is due to surface melting, and that different melting depths are obtained in films with different $T_{m}$ 's. Namely, the non-annealed film has larger melting depth because of its lower $T_{m}$. This larger melting depth in turn allows more crystallinity decrease, as observed in the WAXD. Therefore, the amount of crystallinity decrease is a bulk phenomenon and can be explained by the melting depth in the film.

\section{Chemical Modifications}

Modifications of Chemical Bonds XPS measurements are performed to investigate the chemical modifications on the film surface. The $\mathrm{O}_{1 \mathrm{~s}}$ and $\mathrm{C}_{1 \mathrm{~s}}$ spectra are recorded to determine the amount of $\mathrm{O}$ and $\mathrm{C}$ elements as well as the possible bond breakage. The $\mathrm{C}_{1 \mathrm{~s}}$ spectra of the film with high radical mobility before and after laser treatment are given in Figs. 7(a) to 7(c). The profile is composed of three peaks, which come from the three kinds of carbon groups in the structure: the $\mathrm{C}-\mathrm{H}$ groups, the $\mathrm{C}-\mathrm{O}$ groups in the backbone, and the $\mathrm{O}-\mathrm{C}=\mathrm{O}$ groups in the ester bonds. 
The three groups have binding energies of 285, 287, and $289 \mathrm{eV}$, and contribute to Peak 1, Peak 2, and Peak 3, respectively [34]. The results show that the spectra do not have appreciable change until a high fluence is utilized.

The resolved $\mathrm{C}_{1 \mathrm{~s}}$ spectra are also given in Fig. 7. The areas under the three resolved peaks are calculated and plotted as a function of laser fluence in Fig. 8 for the film with high radical mobility. The results for the film with low radical mobility are similar except that the peak areas change at a lower extent. It is also observed that the peak areas change when the laser

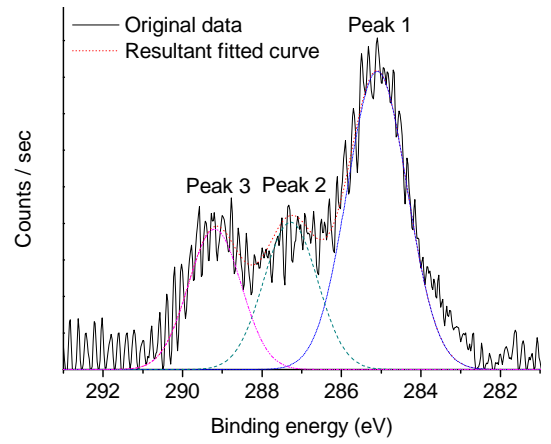

(a)

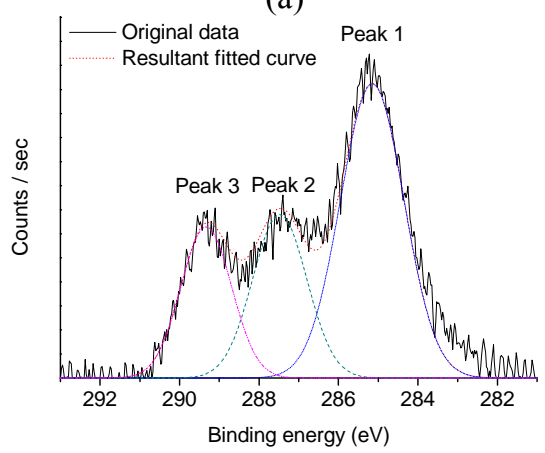

(b)

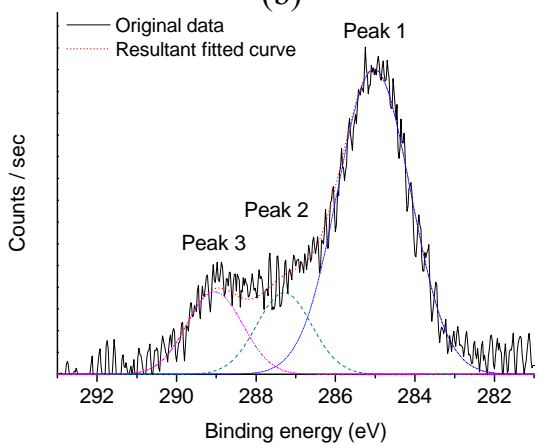

(c)

Figure 7: Typical $\mathrm{C}_{1 \mathrm{~s}}$ XPS spectra and the resolved peaks of the film before and after laser treatment. The figures show the results of the film with high radical mobility (a) before laser treatment, and treated with fluences of (b) $2.5 \mathrm{~J} / \mathrm{cm}^{2}$ and (c) 2.6 $\mathrm{J} / \mathrm{cm}^{2}$. fluence reaches a certain level, which $2.6 \mathrm{~J} / \mathrm{cm}^{2}$ and $2.65 \mathrm{~J} / \mathrm{cm}^{2}$ for the film with high and low radical mobility, respectively. The excimer laser used in this study generates photons with 248-nm wavelength, whose energy is $5.0 \mathrm{eV}$. The chemical bond energies in PLLA are $3.6 \mathrm{eV}$ for the $\mathrm{C}-\mathrm{C}$ bonds, $3.8 \mathrm{eV}$ for the $\mathrm{C}-\mathrm{O}$ bonds, $7.5 \mathrm{eV}$ for the $\mathrm{C}=\mathrm{O}$ bonds, and $4.3 \mathrm{eV}$ for the $\mathrm{C}-\mathrm{H}$ bonds. Since the photochemical reactions occur whenever the energy of a single photon exceeds the dissociation energy, chemical bond breakage occurs even with low laser fluence. The threshold behavior shown in the XPS results, thus, suggests that low-fluence laser treatment induces insignificant amount of chemical modifications which do not reflect in the measurements [9].

Figures 8 and 9 show that, as the chemical modifications occur, the areas under Peaks 2 and 3, as well as the $\mathrm{O} / \mathrm{C}$ ratio, decrease. This demonstrates that the chemical modifications are carried out through the loss and/or breakage of the $\mathrm{C}-\mathrm{O}$ groups in the backbone and the $\mathrm{O}-\mathrm{C}=\mathrm{O}$ groups in the ester bonds,

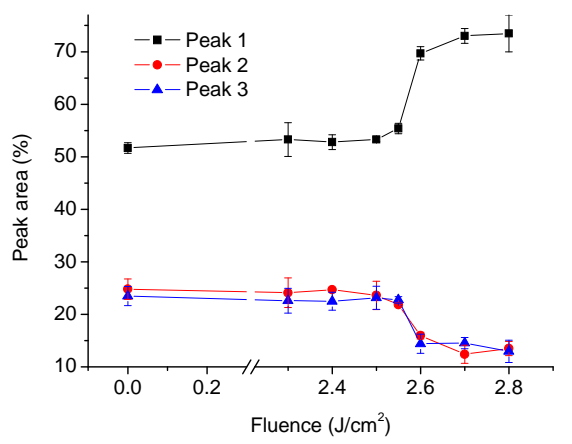

Figure 8: Area percentage of three resolved peaks in the $C_{1 s}$ XPS spectra of the film with high radical mobility.

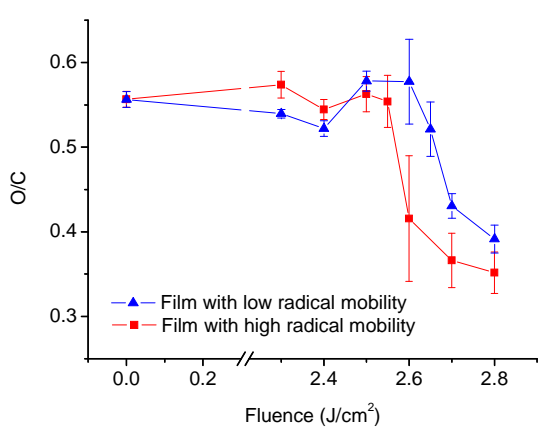

Figure 9: Ratio of the $\mathrm{O}_{1 \mathrm{~s}}$ peak area to the $\mathrm{C}_{1 \mathrm{~s}}$ peak area derived from the XPS spectra for the films with high and low mobility. 
which account for the decrease of Peaks 2 and 3, respectively. The decrease of $\mathrm{O} / \mathrm{C}$ ratio suggests the loss of oxygen atoms. Since bubbles exist on the laser treated films with appreciable chemical modifications, and bubbles are composed of small gaseous products from chemical decomposition, it is believed that a portion of the oxygen atoms emit from the film in form of $\mathrm{CO}$ and $\mathrm{CO}_{2}$.

It is also noticed that the peak area ratio does not agree with the theoretical value, 1:1:1. The peak area $I$ can be determined through the equation

$$
I=K_{0} N_{a} \sigma_{c} \lambda_{p} A_{s} T_{a}
$$

where $K_{0}$ is the instrument constant, $N_{a}$ is the atomic concentration, $\sigma_{c}$ is the photoionization cross section for an element, $\lambda_{p}$ is the inelastic mean-free path length for photoelectrons, $A_{s}$ is the area of the sample, and $T_{a}$ is the analyzer transmission function. Atomic concentration $N_{a}$ can be the reason which causes the deviation from the theoretical value. Larger area of Peak 1 suggests a higher concentration of the side group $\mathrm{CH}_{3}$ on the surface than the other two groups of carbon in the backbone. Since a monolayer of atom on the surface can be detected by XPS, it is believed that the side group is more abundant on the top surface. This could be due to the well-aligned crystalline chains in the ordered lamellae on the spherulitic surface. The spherulites are composed of lamellae, and the spherulitic surface is formed by the lateral surfaces of lamellae. The side groups protrude from crystalline chains may be the outmost atoms on the lateral surfaces of lamellae and may thus form a monolayer on the surface of spherulites. Therefore, in the ordered structure the side group may have higher concentration on the film surface. The explanation is supported by a separated XPS measurement on an amorphous PLLA film made by spin coating with procedures detailed in [29]. The results of both area ratio and atomic ratio (not shown) agree with the theoretical values, since the distribution of the three groups of carbons is more uniform in a less ordered structure.

Effect of Radical Mobility on the Amount of Chemical Modifications The amount of chemical modifications in the films with high and low radical mobility can be revealed by the XPS results, Figs. 8 and 9, and is numerically investigated via simulating the number density of the $\mathrm{CH}_{3} \mathrm{CHCOO}$ units connecting at their ester group with other units. The simulation is generated for the film surface and thus comparable with the XPS results. Figures 8 and 9 show that appreciable chemical modifications occur at a lower fluence for the film with high radical mobility (2.6 $\mathrm{J} / \mathrm{cm}^{2}$ ) and higher fluence for the film with low radical mobility $\left(2.65 \mathrm{~J} / \mathrm{cm}^{2}\right)$. Under the same fluence, the peak areas and the $\mathrm{O} / \mathrm{C}$ ratios also change at a larger extent for the film with high radical mobility. Simulation results demonstrate the same trend. Figure 11 gives the number density of the $\mathrm{CH}_{3} \mathrm{CHCOO}$ units connecting at their ester group with other units for the film with high radical mobility. It shows that higher fluence causes larger amount of chemical modifications. Similar results are obtained for the film with low radical mobility. The final number density is plotted as a function of fluence as given in Fig. 6, which shows that the film with high radical mobility has more chemical modifications. The reason is because radicals with high mobility tend to react with other free radicals and generate decomposed products. This is known as the cage effect, which states that the free radicals generated by the dissociation of molecules are unable to move apart due to the existence of surrounding molecules, with the result that the dissociation products may recombine and return to the initial state. To react with each other it is required that the free radicals meet in a single cage, which is the volume in which the probability of reaction is significantly higher. The cage radius is affected by radical mobility, which is higher in a high temperature or the molten state. The cage radius can reach several tens of angstroms at a temperature close to the melting temperature [35]. Due to large cage radius, the radicals have high possibility to react with other radicals within the cage. When the radical mobility is hindered, such as in the crystalline domain, the effective cage radius decreases to the dimension of the elementary crystal unit cell, and the number of active radicals in the cage is much less. The requisite role for reaction can be played by radical diffusion from cage to cage. However, the diffusion is slow in a polymer matrix with a diffusion coefficient on the order of $10^{-15}$ to $10^{-18} \mathrm{~cm}^{2} / \mathrm{s}$. This slow diffusion process leads to a high probability of free radical pair recombination in the cage, which is dominant in the crystalline domain. This accounts for the phenomenon that it requires higher laser fluence to cause chemical modifications in the high-radial-mobility film and its larger amount of chemical modifications.

Figure 6 also shows that chemical modifications always occur even a small fluence is used. Namely, no chemical modification threshold exists, which agrees with photochemical decomposition theory. The threshold observed in the XPS measurements, Figs. 7-9, is due to insignificant amount of chemical modifications. The amount of chemical modifications 
become appreciable after the fluence increases to a certain level, and then can be detected.

\section{Conclusions}

PLLA crystallinity change and chemical modifications under excimer laser irradiation are experimentally and numerically investigated. Crystallinity decrease due to laser melting is demonstrated, and the effects of radical mobility on chemical modifications are evaluated. The amount of chemical modifications is determined by the free radical mobility, which is decreased by increasing the degree of crystallinity through annealing before laser treatment. Both WAXD and simulation results show that the decrease of crystallinity initiates at the same fluence for both films with high and low radical mobility. According to the XPS measurements and simulation, the film with low radical mobility has a smaller amount of chemical modifications, and larger fluences are required to cause appreciable chemical modifications. Films with smaller radical mobility have a larger working window, in which the surface melting occurs while the chemical modifications are limited. A larger working window increases the applicability of surface treatment by the excimer laser.

\section{Acknowledgements}

Financial support from NSF under CMMI-1030536 is acknowledged. WAXD and XPS measurements were carried out at MRSEC, Columbia University.

\section{References}

[1] Amass, W., Amass, A. \& Tighe, B. (1998) A review of biodegradable polymers: uses, current developments in the synthesis and characterization of biodegradable polyesters, blends of biodegradable polymers and recent advances in biodegradation studies, Polymer International 47, 89-144.

[2] Zhao, Y., Fu, J., Ng, D.K.P. \& Wu, C. (2004) Formation and degradation of poly(D,L-lactide) nanoparticles and their potential application as controllable releasing devices, Macromolecular Bioscience 4, 901-906.

[3] Miller, R.A., Brady, J.M. \& Cutright, D.E. (1977) Degradation rates of oral resorbable implants (polylactates and polyglycolates): rate modification with changes in PLA/PGA copolymer ratios, J. Biomed. Mater. Res. 11, 711-719.

[4] Tsuji, H. (2002) Autocatalytic hydrolysis of amorphous-made polylactides: effects of L-lactide content, tacticity, and enantiomeric polymer blending, Polymer 43, 1789-1796.

[5] Nampoothiri, K.M., Nair, N.R. \& John, R.P. (2010) An overview of the recent developments in polylactide (PLA) research, Bioresource Technology 101, 8493-8501.

[6] Tsuji, H. \& Ikada, Y. (1998) Properties and morphology of poly(L-lactide). II. hydrolysis in alkaline solution, J. Polym. Sci. A: Polym. Chem. 36, 59-66.

[7] Tsuji, H. \& Ikada, Y. (1995) Properties and morphology of poly(L-lactide). 1. annealing condition effects on properties and morphologies of poly(L-lactide), Polymer 36(14), 2709-2716.

[8] Bhatla, A. \& Yao, Y.L. (2009) Effect of laser surface modification on the crystallinity of poly(L-lactic acid), Journal of Manufacturing Science and Engineering 131, 051004

[9] Lazare, S. \& Benet, P. (1993) Surface amorphization of mylar films with the excimer laser radiation above and below ablation threshold: ellipsometric measurements, J. Appl. Phys. 74(8), 4953-4957.

[10] Dunn, D.S. \& Ouderkirk, A.J. (1990) Chemical and physical properties of laser-modified polymers, Macromolecules 23, 770-774.

[11] Watenabe, H., Takata, T. \& Tsuge, M. (1993) Polymer surface modification due to excimer laser radiation-chemical and physical changes in the surface structure of poly(ethylene terephthalate), Polymer International 31, 247-254.

[12] Belyayev, O.F. (1988) Mechanism of melting of oriented polymers, Polymer Science U. S. S. R. 30(11), 2545-2552

[13] Yamamoto, T. (2010) Molecular dynamics of reversible and irreversible melting in chain-folded crystals of short polyethylene-like polymer, Macromolecules 43, 9384-9393.

[14] Cormia, R.L., Price, F.P. \& Turnbull D. (1962) Kinetics of crystal nucleation in polyethylene, Journal of Chemical Physics 37(6), 1333-1340.

[15] Hoffman, J.D. \& Weeks, J.J. (1962) Melting process and the equilibrium melting temperature of polychlorotrifluoroethylene, Journal of Research of the National Bureau of Standards-A. Physics and Chemistry 66A(1), 13-28.

[16] Mitomo, H. (1988) Correspondence of lamellar thickness to melting point of nylon-6,6 single crystal, Polymer 29, 1635-1642. 
[17] Di Lorenzo, M.L. (2005) Crystallization behavior of poly(L-lactic acid), European Polymer Journal 41, 569-575.

[18] Lazare, S. \& Granier, V. (1989) Ultraviolet laser photoablation of polymers: a review and recent results, Laser Chem. 10, 25-40.

[19] Janorkar, A.V., Metters, A.T. \& Hirt, D.E. (2007) Degradation of poly(L-lactide) films under ultraviolet-induced photografting and sterilization conditions, Journal of Applied Polymer Science 106, 1042-1047.

[20] Inagaki, N., Narushima, K., Tsutsui, Y. \& Ohyama, Y. (2002) Surface modification and degradation of poly(lactic acid) films by Ar-plasma, Journal of Adhesion Science and Technology 16(8), 1041-1054.

[21] Schneider, S., Richter, F. \& Brem, B. (1998) The photodegradation of poly(2,6-dimethyl-1,4-phenylene oxide)-a flash photolysis study of polymer and model compounds, Polymer Degradation and Stability 61, 543-464.

[22] Rebollar, E., Bounos, G., Oujja, M., Georgiou, S. \& Castillejo, M. (2006) Effect of molecular weight on the morphological modifications induced by UV laser ablation of doped polymers, J. Physical Chemistry B $110,16452-16458$.

[23] Efthimiopoulos, T., Kiagias, H., Christoulakis, S. \& Merlemis N. (2008) Bubble creation and collapse during excimer laser ablation of weak absorbing polymers, Applied Surface Science 254, 5626-5630

[24] Dan, E. \& Guillet, J.E., 1973, Photochemistry of ketone polymers. $\mathrm{X}$. chain scission reactions in the solid state, Macromolecules 6(2), 230-235.

[25] Aleksandrov, A.P., Kitai, M.S. \& Varbanskaya, R.A. (1988) Photochemical autocatalytic propagation reaction of polyene sequences in photodegradation of polyvinyl chloride, Polymer Science U.S.S.R. 30(8), 1687-1696.

[26] Ohki, Y., Hirai, N., Fuse, N., Tanaka, T., Kohtoh, M. \& Okabe, S. (2007) Search for adequate biodegradable polymer as an eco-friendly electrical insulating material, in Proceedings of the International Symposium on EcoTopia Science, Nagoya, Japan, 488-493.

[27] Bityurin, N., Luk'yanchuk, B.S., Hong, M.H. \& Chong, T.C. (2003) Models for laser ablation of polymers, Chemical Review 103, 519-552.

[28] Astrath, N.G.C., Astrath, F.B.G., Shen, J., Zhou, J., Michaelian, K.H., Fairbridge, C., Malacarne, L.C., Pedreira, P.R.B., Santoro, P.A. \& Baesso, M.L. (2009)
Arrhenius behavior of hydrocarbon fuel photochemical reaction rates by thermal lens spectroscopy, Applied Physics Letters 95, 191902.

[29] Currie, J.A., Bohm, G.G.A. \& Dole, M. (1969) Calorimetric detection on free radicals in irradiated polyethylene, Polymer Letters 7, 535-539.

[30] Schindler, A. \& Harper, D. (1979) Polylactide. II. viscosity-molecular weight relationships and unperturbed chain dimensions, J. Polym. Sci.: Polym. Chem. Ed. 17, 2593-2599.

[31] Hsu, S.-T. \& Yao, Y.L. (2011) Effect of film formation method and annealing on crystallinity of poly (L-lactic acid) films, in Proceedings of the International Manufacturing Science and Engineering Conference, Oregon, USA, MSEC2011-50205.

[32] Srinivasan, R. (1993) Pulsed ultraviolet laser interactions with organic polymers dependence of mechanism upon laser power, Polymer Degradation and Stability 43, 101-107.

[33] Alexander, L.E. (1969) X-Ray Diffraction Methods in Polymer Science, John Wiley \& Sons, New York.

[34] Beamson, G. \& Briggs, D. (1992) High Resolution XPS of Organic Polymers, John Wiley \& Sons, Chichester.

[35] Butiagin, P.J. (1972) The decay of free radicals in polymer media, in Proceedings of the International Conference on Chemical Transformations of Polymers, Bratislava, Czechoslovakia, 57-76.

\section{Meet the Authors}

Shan-Ting Hsu received his B.S. and M.S. from National Taiwan University. $\mathrm{He}$ is currently a doctoral student at the Manufacturing Research Laboratory at Columbia University.

Huade Tan received his B.S. from Boston University and M.S. from the University of Notre Dame. He is currently a doctoral student at the Manufacturing Research Laboratory at Columbia University.

Dr. Y. Lawrence Yao is currently a professor of Columbia University's Mechanical Engineering Department and director of the Manufacturing Research Laboratory. He received his Ph.D. from the University of Wisconsin-Madison in 1988. 